\title{
The Use of Modern Contraceptives Among Women of Child Bearing Age Attending MCH/FP Clinic at Uasin Gishu Sub-County Hospital, Uasin-Gishu County, Kenya
}

\author{
Robert M. Kei, Taratisio Ndwiga, Stephen Okong'o \\ Department of Environmental Health, School of Public health, Moi University, Eldoret Kenya
}

Email address:

taratisiondwiga@yahoo.com (T. Ndwiga)

To cite this article:

Robert M. Kei, Taratisio Ndwiga, Stephen Okong'o. The Use of Modern Contraceptives Among Women of Child Bearing Age Attending MCH/FP Clinic at Uasin Gishu Sub-County Hospital, Uasin-Gishu County, Kenya. Science Journal of Public Health.

Vol. 3, No. 4, 2015, pp. 500-507. doi: 10.11648/j.sjph.20150304.17

\begin{abstract}
Introduction: Family planning is a practice of controlling on when to have children through the use of contraceptives. Contraceptive use in developing countries has decreased the maternal deaths by $40 \%$ and could prevent $70 \%$ if the full demand for birth control is met. Problem statement: Despite serious campaign programs to sensitize women of reproductive age to take up contraception, there is still a rise in population and other problems such as rise in the number of street children which negatively impact on economy and available resources. Objectives: To identify the types of contraceptives used, to determine the perception of women on contraceptive use, to determine the factors associated with contraceptive use and to assess the effectiveness of the different types of the contraceptives. Justification: The study sought to determine the extent to which each contraceptive method was known and used, the effectiveness and factors that determined the use of contraceptives in women of child bearing age. Methodology: The study population included all women of child bearing age (15-49 years) attending MCH/FP clinic at Uasin Gishu Sub-County Hospital. A cross sectional descriptive survey was the design and Systematic random sampling was used to select respondent. Chi-square test was used to measure the strength of associations between variables where a $p$-value of $=$ or $<0.05$ was considered to be statistically significant. Results: The study showed that a large percentage of the respondents had knowledge on family planning methods with pills being the most known, the most commonly used method of contraception was injectables and among the methods used condom had the least complications. Conclusion: The contraceptives available were injectables, pills, male and female condoms, implants, IUCD and respondents also had knowledge on Natural Family planning (NFP), among these injectable and pills were mostly preferred. Recommendations: The health workers within the $\mathrm{MCH}$ should not limit the health education only on the methods available in the facility but should include all family planning methods.
\end{abstract}

Keywords: Family Planning, Natural Family Planning, Contraception, Intra-Uterine Contraceptive Device, Mother Child Health

\section{Introduction}

Rapid population increase in the world especially 3rd world countries has become a major concern which requires mitigation. High population leads to scramble for resources which are scarce. It is also difficult to plan how to divide national resources and has become a cause for decline in economy. High population has its advantages like increase of human labor but when it is more than the required it poses dangers of increase in crimes as a source of livelihood as well as increases in the number of street children hence the need to curb population growth for better economic management.

Contraceptive methods can be grouped into modern contraceptives which include; hormonal, sterilization and barrier methods while the traditional ones include withdrawal, rhythm and cycle methods.

Unwanted pregnancies are a major public health problem for both developing and developed nations. Unplanned /mistimed pregnancies generally result from ineffective use of contraceptives and result in induced abortions.

Unintended pregnancies remain a major concern in developing countries with 120 million women wanting to postpone child bearing or limit the size of their families, 
(Harrington et al, 2012).

All the same contraceptive use in developing countries has reduced the number of maternal deaths by $44 \%$ (about 270,000 deaths averted in 2008) but could prevent $73 \%$ if the full demand for birth control were met, (Ehlers, 2009).

\section{Problem Statement}

Despite serious campaign programs to sensitize women of reproductive age to take up contraception and provision of contraceptive in public health facilities, there is still a rise in population at a rate of $2.9 \%$ per annum according to the Kenya Bureau of statistics and other problems such as rise in the number of street children which negatively impact on economy and available resources, increased prevalence of sexually transmitted diseases and infections which are costly to treat, unwanted pregnancies leading to rise of death during abortions.

The use of modern contraceptives is still low at $37.6 \%$ raising the need for more efforts to make modern contraceptive use more widespread among women in urban Kenya (Teresa et al, 2011).

The population in Kenya has been increasing at a rate of $2.9 \%$ per annum according to Kenya Bureau of Statistics. This has come along with serious economic and social challenges including the stretching of the various social facilities such as schools, hospitals and housing. The study sought to determine the extent to which each contraceptive method is known and used, the effectiveness and factors that determine use of contraceptives in women of child bearing age.

The aim was to assess the utilization of contraceptives among women of child bearing age attending MCH/FP clinic at Uasin-Gishu Sub-County Hospital, with to identify types of contraceptives available, to identify the types of contraceptives used, to determine the perception of women on contraceptive use and to determine the factors associated with contraceptive use.

\section{Literature Review}

In spite of efforts towards fertility control, there remains a substantial proportion of women in the reproductive age group (15-49 years) who are not using FP methods even though they do not want a pregnancy. This explains the fact that about two-thirds of women of reproductive age (15-49 years) are at risk of unintended pregnancy, (Ehlers, 2009). This results from either non-use or ineffective use of contraceptive methods.

Use of modern family planning varies by residence and province. Modern methods are used by $47 \%$ of married women in urban areas, compared with $37 \%$ of women in rural areas. Modern contraceptive use ranges from a low of $4 \%$ of married women in North Eastern province to a high of $63 \%$ in Central province. (KDHS, 2008/09).

In a study done in Kibera slums, most non-users (14.3\% of the study sample) were aged between 15-19 years, while the highest rate of usage was recorded amongst age-group 30-34 years $(8.3 \%$ of the study sample. (Murerwa, 2012), the study also recommends interventions, programs and policies to increase contraceptive utilization. This is intended to serve ultimately as a guide for population and demographic planning.

In another study also carried out in Kenya on the use of modern contraceptives, it was clearly indicated that in contrast to the use of modern birth control methods, the majority of the respondents $(57 \%)$ used the traditional birth control methods, while $13.3 \%$ of the respondents did not use any form of birth control methods, (Nwachukwu, et al, 2008).

Although contraceptive use is high $(71.8 \%)$ in slum and non-slum areas, use of modern contraceptives is still low at $37.6 \%$. raising the need for more efforts to make modern contraceptive use more widespread among women in urban Kenya (Teresa, et al 2011).

\section{Factors Influencing Use of Contraceptives}

\subsection{Level of Education}

Several other studies demonstrated that the lower the level of education the woman has, the less likely she is to use contraceptives. The incidence of unwanted pregnancies is high amongst the less educated women (Fikree, et al 2001). It is also clear that the uneducated woman is unlikely to find a worthwhile job, so her hope for survival is to find a husband who will support her. She then performs her household duties, whilst the man decides on the size of the family and whether she can use contraceptives or not (Ehlers, 2009). Education helps women to access and use the clinics properly.

Never having been to school remains a strong predictor of non-contraceptive use. In a contraception study of 883 women in Kericho, $49 \%$ of the women without formal education were found to have never used contraceptives, compared to $31 \%$ who had either primary or secondary education and were using contraceptives (Nazar et al, 2010).

In a study conducted in South Nyanza region (George et al, 2002). It was realized that if a woman is uneducated then she cannot enjoy her reproductive rights. Women with higher education are able to comprehend information about contraceptive methods, and are more aware of their human rights including their reproductive rights. Educated women can initiate the use of contraceptives, constituting the selfcare construct, but uneducated women's inability to access contraceptives belongs to the self-care deficit construct. Making the approach in helping both these groups of women differs.

However the high literacy levels in central province $(88 \%$ of the women are literate in central province) is an attribute to the high use of contraceptives in the region (Wangui, 2012).

There is a gap between awareness and practice of contraception. Despite having knowledge of contraception, compliance is low. Fear of side effects of contraception is one 
significant reason for low utilization (Ambareen, et al, 2011)

In many African cultures prospective husbands pay bridal prize in the form of money or a particular number of cattle to the bride's family. Men, because they have paid bridal prize, could regard their wives as possessions that they have bought. Women end up neither being able to decide on the number of children desired nor about use of contraceptives. In some circumstances the men can claim back his bridal prize if a wife fails to bear children (Ehlers, 2009).

Pretorius (2006) explained some disadvantages South African women face. They are expected to keep up with marital infidelity, emanating from the culture of polygamous marriage. Black South African men believe they have insatiable sexual needs. If a woman cannot bear children in a marriage her husband can easily engage in extramarital affairs and bear children outside marriage, and/or marry additional wives.

In South Africa, black women are expected to use their reproductive ability in order to be accepted by their in-laws and other members of the community (Erasmus et al, 2000). Erasmus also explains that if contraceptives are perceived as rendering women infertile, this poses a serious challenge to effective contraceptive usage. Females are not regarded as real women until they are mothers. Children symbolize a man's wealth and his future insurance. Manliness is mainly judged by the number of children fathered by the man.

In polygamous marriages, the wife with most children is likely to be the husband's favorite wife. Women in this kind of a marriage are not likely to use contraceptives, if they are competing to be the husband's favorite wife with the largest number of children. Sometimes women are required to prove their fertility even before the payment of bridal prize, because children in many African cultures are very important, they give value, meaning, dignity and status to the couple. In these instances even adolescents might not be willing to use contraceptives since they know the values, culture and beliefs about children in their societies (Ehlers, 2009).

\subsection{Contraception Barriers}

The main reasons for non- use of contraceptives were the fear of side effects, husbands' disapproval and the desire for more children, with religion and family setting having a significant association with the use of modern contraceptive methods. (Adeomi et al, 2009)

A study which was conducted in the Baba Dogo urban slum area of Nairobi and Chwele rural sub-location in Bungoma, western Kenya clearly states that some of the important barriers to contraceptive use were identified in couples wishing to space or limit further births. Those barriers included lack of agreement on contraceptive use and on reproductive intentions, husband's attitude on his role as a decision maker, perceived undesirable side effects, distribution and infant mortality, negative traditional practices and desires such as naming relatives and preference for sons as security in old age.

There were also gaps in knowledge on contraceptive methods, fears, rumors and misconception about specific methods and unavailability or poor quality of services in the areas studied. (Kamau et al, 2006).

\subsection{Reasons Leading to Use of Contraceptives}

A study carried out in Uganda (Timothy, et al, 2011) 51\% of the respondents were using family planning services while the remaining $49 \%$ were not. Those using the contraceptives cited various reasons ranging from managing the family to preventing sexually transmitted diseases. Whereas $20 \%$ of the respondents were using family planning to manage the family, 30\% were using family planning for purposes of preventing pregnancy. This result points out that the major reasons why women use contraceptives were to prevent pregnancy and contraction of STIs. On the other hand, nonuse of contraceptives was attributed to infrequent sex, not married, desire for children, cultural norms, pregnancy, religion and lack of support from the partner. In terms of methods, the study reveals that the most commonly used contraceptives were condoms (35\%), pills (33\%), injection (19\%), and IUD (4\%)

It has also been noticed that fears of premature death, financial hardship and prenatal HIV transmission emerged as reasons for HIV patients' desire to delay or cease child bearing, however the uptake of modern contraceptives is constrained by limited access and weak service delivery. (Harrington et al, 2012), in addition, the need for social, economic and health benefits conferred through smaller family size and longer interval between the births of the children increased opportunities of pre-conception counseling and screening.

To avoid the contraction of sexually transmitted infections which increase the risk of preterm birth, low birth weight and still birth Contraception plays a key role in reducing reliance on elective abortions and can avert as many as $13 \%-15 \%$ of the maternal deaths that can result from unsafe abortions. (Tsui et al, 2011). There is also influence from health care providers on their reproductive intentions (Harrington et al, 2012)

Slow growth of population in central province (KNBS 2009 ) is as a result of family planning. Acceptance of family planning information has enabled them to have knowledge on different ways of family planning and therefore manage to keep the intended number of children (Wangui 2012).

Due to good infrastructure in central province most of the women can access health facilities and be advised on the right method to use as well as favorable economic status most of the women in central province can afford a family planning method making it easier for use (Wangui 2012).

Good family planning information and easy access to family planning leads to the use of contraception and hence achieve a much smaller family size that is desirable (Zhushi et al, 2008)

People's decisions about contraceptive use are primarily motivated by the three main purposes of contraception: prevention of pregnancy, prevention of sexually transmitted infections (STI's), and menstrual and fertility regulation. (Julie 2009). 


\subsection{Effectiveness of Contraceptive Use Amongst Women}

Effectiveness is often the most important consideration when a woman chooses a contraceptive method. Many women though don't understand how well various methods protect them against pregnancy and hence usually depend almost entirely on the information they get from their providers who in turn usually explain effectiveness by informing their clients of the pregnancy rates for each method during typical use and during times when it is used consistently and correctly (Family Health International, 2007).

A randomized trial using 461 women in India and Jamaica carried out by Family Health International and Bloomberg School of Public Health (2007) found out that with correct and consistent use of various contraceptive methods, the hormonal based such as implants, progestin only injectables and pills were very effective whereas traditional methods such as Two- Day method, Standard - Day method and withdrawal method were least effective.

\section{Methodology}

The county has six constituencies: Soy, Turbo, Moiben, Ainabkoi, kapseret, Kesses and three administrative SubCounties: Eldoret East, Eldoret West and Wareng, with Eldoret town being the administrative headquarters, according to the 2009 census, Uasin Gishu has a population of $894,179,2.3 \%$ of the nation's population, with 202,291 households and a population density of 269 people per $\mathrm{km} 2$.The age distribution is $0-14$ years $41.5 \%, 15-64$ years $55.7 \%$ and above 65 years $2.9 \%$.

The county has relatively well established infrastructure including Kenya's third international airport. In the health sector, it is served by: dispensaries (88), health centers (23), sub-County hospitals (2), County (1), National hospital (1) and private facilities.

Uasin-Gishu Sub-County Hospital mainly serves people from the different estates in Eldoret municipality and the neighboring areas. Services offered at the hospital include: Maternal Child Health (MCH)/Family Planning (FP), CCC (Comprehensive care Centre), Maternity and other reproductive services, Curative Adults and Pediatric outpatient services. Other specialized services include Dental Orthopedic, Ophthalmology, Occupational therapy and Physiotherapy services.

Within the MCH/FP clinic, services such as vaccination, nutritional counseling, health education and family planning are offered. In family planning department, contraceptives such as implants, injectables, IUCDs and oral contraceptives are provided depending on factors such as side effects, cost and preference. The study population included all women of child bearing age (15-49 years) attending $\mathrm{MCH} / \mathrm{FP}$ clinic at Uasin Gishu Sub-County Hospital. A cross sectional survey was carried out within two months (September and October) at the study area using structured questionnaires. It utilized both qualitative and quantitative data collection methods.
Uasin Gishu Sub-County Hospital has an average of 1100 women visiting the $\mathrm{MCH} / \mathrm{FP}$ in a month.

A sample size of 421 persons was determined by use of Fisher et al formula, 1998.

Systematic random sampling was used to select respondents, where the first respondent was the first person to be attended to on that day of interview. Thereafter the other respondents were chosen at an interval of five, the interval of five was derived from dividing the hospital's approximation of the expected attendance for the two months of the research period by the sample size. This was repeated in the subsequent days until a sample size of 421 respondents was attained.

All women of child bearing age between the ages 15-49 years, attending MCH/FP clinic at Uasin Gishu Sub-County Hospital and who were using contraceptives were allowed to participate.

Data was collected using structured questionnaires and interviews guides. A pilot study was carried out at Huruma Sub-County hospital to pre-test the instruments that were used with an aim of testing reliability thereafter appropriate revision and adjustment was done. The questionnaire was tested on twenty respondents. Data was collected using interview schedule questionnaires then reviewed to identify anomalies such as omissions that were are corrected before leaving the field.

\subsection{Data Analysis}

Data from all questionnaires were coded and entered in the Statistical Package for social scientists (SPSS) Version 20, which was used both as a data base and for data analysis. Frequency distribution and analysis of the data was produced and the descriptive statistics was used for tabular and graphic presentation of the findings. Chi-square test was used to measure the strength of associations between variables where a $p$-value of $=$ or $<0.05$ was considered to be statistically significant.

\subsection{Ethical Considerations}

The nature and purpose of the research was explained to the respondents by the researchers. Permission and approval from Uasin-Gishu Sub-County Hospital to carry out the research was sought from the hospital management. Participation from the respondents was voluntary. Confidentiality and personal integrity was safeguarded.

\section{Results}

Socio-demographic characteristics of the pregnant women attending antenatal clinic in Uasin-Gishu Sub County hospital $(\mathrm{N}=421)$

$\begin{array}{ll}\text { Age of the respondents } & N(\%) \\ 15-25 & 199(47 \%) \\ 26-35 & 189(45 \%) \\ 36-45 & 29(7 \%) \\ 46-49 & 4(1 \%)\end{array}$


Marital Status of the respondents

Married

Single

Separated

Widowed

Education level of the respondents

Primary

Secondary

Tertiary

Occupation of the respondents

Unemployed

Self-employed

Employed in private sector

Employed in public sector

Knowledge On Family Planning

Methods known by the respondents

Pills

Injectables

Male Condom

Implants

IUCD

Female condom

Natural Methods

Tubal Ligation

Hysterectomy

Spermicides

Methods used by the respondents

Injectables

Pills

Male Condom

Implants

Natural Methods

$333(79 \%)$

$76(18 \%)$

$8(2 \%)$

$4(1 \%)$

$96(22.8 \%)$

$145(34.4 \%)$

$180(42.8 \%)$

$208(49.4 \%)$

$90(21.4 \%)$

$75(17.8 \%)$

$48(11.2 \%)$

396 (94\%)

$375(89 \%)$

$370(88 \%)$

$320(76 \%)$

$282(67 \%)$

$257(61 \%)$

$227(54 \%)$

199 (47\%)

$135(32 \%)$

$118(28 \%)$

$160(38 \%)$

$80(19 \%)$

$63(15 \%)$

$55(13 \%)$

$21(5 \%)$

$16(4 \%)$

$15(3.6 \%)$

$8(2 \%)$

$3(0.4 \%)$

$0(0 \%)$

Hysterectomy

Period for using current Contraceptive

Less than 6 months

$139(33 \%)$

$101(24 \%)$

$67(16 \%)$

$114(27 \%)$

1-2 years

$408(97 \%)$

$13(3 \%)$

No

Facing Challenges while using Contraceptives

Yes

$63(15 \%)$

No

$358(85 \%)$

Complications experienced

Menstrual complication

$181(44 \%)$

Backache

$84(20 \%)$

Weight gain

$51(12 \%)$

$46(11 \%)$

eadache

$21(5 \%)$

$21(5 \%)$

$13(3 \%)$

Lower limb pain

Secondary Infertility
Partner support on choosing Family Planning method

$\begin{array}{ll}\text { Yes } & 345(82 \%) \\ \text { No } & 76(18 \%)\end{array}$

Table 1. Correlation between age and Number of children.

\begin{tabular}{lllllll}
\hline \multirow{2}{*}{ AGE } & \multicolumn{7}{l}{ Number Of Children } \\
\cline { 2 - 7 } & $\mathbf{1}$ & $\mathbf{2}$ & $\mathbf{3}$ & $\mathbf{4}$ & 5 AND ABOVE & NONE \\
\hline $15-25$ & 109 & 45 & 9 & 3 & 3 & 29 \\
$26-35$ & 43 & 77 & 39 & 20 & 5 & 5 \\
$36-45$ & 2 & 7 & 10 & 7 & 3 & 2 \\
$46-49$ & 0 & 0 & 2 & 1 & 0 & 0 \\
TOTALS & 154 & 129 & 60 & 31 & 11 & 36 \\
\hline
\end{tabular}

$\mathrm{X}^{2}=1.312, \mathrm{df}=18, \mathrm{PV}=0.000$

Majority of the respondents had between 1 and 2 children. There was correlation between age and number of children $\left(\mathrm{X}^{2}=1.312, \mathrm{df}=18, \mathrm{PV}=\right.$ $0.000)$.

\section{Discussion}

\subsection{Knowledge on Contraceptives}

Uasin Gishu Sub-County hospital MCH/FP clinic serves a wide range of clients whose level of education ranges from primary to tertiary and this is because it's located in an urban area. The respondents interviewed in the study were educated from primary, secondary and tertiary constituted $22.8 \%$, $34.4 \%$, and $42.8 \%$ respectively but despite the difference in level of education most $98.8 \%$ still had knowledge on family planning methods.

This concurs with previous studies by Fikree et.al 2001 and Nazar et.al 2010 in their study in Kericho in which they found that most women in their studies without formal education or with lower level of education had little knowledge on contraceptives and thus less utilization. Our study also concurred with another study in south Nyanza region by George et al, 2002 who found out that women with higher level of education had more knowledge and understanding of contraceptives. This is quite consistent with this case as illustrated in a case in which some of our respondents with primary education knew about condoms but not as a contraceptive but as a method of preventing sexually transmitted diseases and HIV yet the former is also true.

It was also noted that despite the level of education playing a major role in extent of knowledge on contraceptives, the knowledge seemed to be limited to what was offered at the facility with many respondents regardless of their level of education did not know the contraception methods not available at the facility e.g. Hysterectomy and spermicides were known by $32 \%$ and $28 \%$ of respondents respectively. This was attributed to the health workers at the facility limiting their talks and teachings on contraceptives to those offered at the facility therefore challenging the perception that respondents having higher levels of education knew more on contraceptives compared to the rest with lower levels of education.

\subsection{Use of Contraceptives in Relation to Age}

The use of contraceptives among young married women, 
(aged 15-25) and older married women (aged 26-49) is slightly different as reported by $47 \%$ and $53 \%$ of the respondents respectively. The reason being that majority of the young married women still had desire for more children. A major factor that played a role in whether one used any form of contraceptive was based on the education level of an individual, those women who had attained at least secondary education had adequate knowledge on contraceptives which determines whether someone will use them or not. It is apparent to presume that the older women had gone through more education levels as corresponding to their age.

The most (38\%) commonly ever used methods among all women are injectables irrespective of whether they are in the younger age group or not, this corresponds with studies of KDHS 2008/09. This finding is different from that done in Uganda (Timothy, et al, 2011) whereby condoms were the main method of contraception used. Condoms were majorly used by the young women $10 \%$ as compared to $5 \%$ of the older women which is as twice as much. However with the use of implants and IUCDs, use among the older women was prevalent with percentages of $9 \%$ each.

The high usage of contraceptives has played a major role in regulating family sizes as a majority of the respondents had no more than two children at the period of study (See table 1).

\subsection{Challenges with the Use of Contraceptives}

Majority of the respondents $(97 \%)$ reported not to face any challenge with regard to the use of contraceptives while $3 \%$ faced various challenges, these included; condom bursting during sexual intercourse because the partner did not know how to use it, the condom not being of the right size or the partner refusal to use condom. Some procedures were cited to be costly such as implants which are usually around $200 \mathrm{kshs}$ hence some of the respondents said they were not able to afford therefore ended up using the cheaper contraceptives. The contraceptives needed by some respondents were not available at the hospital hence they ended up settling for the ones available, lack of tangible knowledge on some contraceptives such as tubal ligation, hysterectomy ,Spermicides where we found out that majority of the respondents had very little or no knowledge on them, this could be attributed to those contraceptives not being available at that hospital hence the health care providers found it difficult and unnecessary to teach something they did not have. Those respondents using pills cited various challenges such as forgetting to take the pills on time and it being tiresome. Religious background where there were certain beliefs discouraging the use of contraceptives usually observed among Catholics, hence the women ended up using the contraceptives without the knowledge and approval of their spouses. The respondents that were using natural method of family planning that is, counting of safe days also encountered a challenge of it being difficult to follow up on the safe days because the periods could sometimes be irregular.

Some of the barriers found concurred with those of a study which was conducted in the Baba Dogo urban slum area of Nairobi and Chwele rural sub-location in Bungoma, western Kenya clearly states that some of the important barriers to contraceptive use were identified in couples wishing to space or limit further births. Those barriers included lack of agreement on contraceptive use and on reproductive intentions and husband's attitude on his role as a decision maker.

\subsection{Complications Arising from the use of Contraceptives}

It was noted that a significant $36.2 \%$ of respondents experienced complications resulting from the contraceptive method they were using. Menstrual problems, weight gain, weight loss, headache, dizziness and backache were among the reported complications with menstrual problems topping with the highest frequency. Menstrual complications such as irregular periods and increased bleeding were caused by hormonal imbalance in the body, since hormonal contraceptives contain estrogen and progesterone hence increasing the levels of hormones in the body thus causing complications.

It was noted that injectables were the most used contraceptive method and which also presented complications on $18.6 \%$ of its users. "Depo provera" is an injectable drug that prevents pregnancy for three months at a time. It is a choice for women who want safe, reliable and reversible contraception. The drug contains a female hormone (progesterone) that stops an egg from being released by the ovary. Those using Injectables had complications such as backache, secondary infertility, weight gain, dizziness and menstrual complications which were more common. Oral pills were the second widely used contraceptive with $8.3 \%$ of users experiencing problems. The women who were currently using pills had complications such as backache, headache, weight gain, lower limb pain, loss of appetite and menstrual complications being common. Those who were using implants also had certain complications like backache, menstrual complications, headaches, weight gain and loss of appetite; however those on IUCD had only backache and menstrual complications which were less likely to occur.

There were less or no complications among those who reported to be using condoms, spermicides, tubal ligation and hysterectomy.

Most of the complications resulted from use of injectables and oral pills, women planning to use contraceptive methods should be enlightened on other methods which are nonhormonal and with less side effects. These methods identified on this research included use of IUCD, tubal ligation, condoms among others.

Generally, women who used hormonal contraceptive methods had a higher likelihood of developing complications.

\subsection{Effectiveness of Contraceptives}

According to the study, majority of the respondents (97\%) reported that the contraceptives they were using were 
effective. This shows that three in every hundred in the study became pregnant after the use of a modern contraceptive method.

The high rate of effectiveness on modern contraceptives has led to its high use among women of child bearing age, thus they attain their desired number of children and determine the spacing of children. In addition to controlling pregnancies, women have also protected themselves against STIs by use of condoms, reduced the need for unsafe abortions and unnecessary maternal deaths. This is essential to securing the well-being and autonomy of women, while supporting the health and development of communities.

Those that found the contraceptives to be ineffective cited various reasons which were specific to the type of contraceptive method being used.

In the case of those using pills, the continuous and consistent use of pills as directed by the health practitioner was cited as a challenge among women using this form of contraception. In other cases, women took the pills only when their partners were around, which is not how the contraceptive works. Reasons for this poor use could be due to poor communication during administration where the method is not explained well, a language barrier between the health practitioner and the patient or the misinterpretation of the instruction on use of the contraceptive. This could either be the fault of the health practitioner or the patient. This caused women to skip a day or even days, during the use of the contraceptive, reducing the efficiency of the method in preventing pregnancies.

In the Natural Family Planning (NFP) method, women had difficulty in identifying their fertile days mainly caused by irregular menstrual periods and little understanding of the calculation of safe days.

Barrier methods like male and female condoms were reported to burst or slip off during use. These were major problems as the removal of the barrier renders the method ineffective. This is caused by poor knowledge on use of barrier method or poor quality of material used to make the contraceptive.

Most of the respondents found pills, injectables and implants to be more effective than the natural method which concurs with literature in a study done in India by family health international and Bloomberg School of Public Health (2007) which found implants, injectables and pills to be very effective.

\section{Conclusion and Recommendations}

\subsection{Conclusion}

It was found out that the contraceptives available in UasinGishu hospital were injectables, pills, male and female condoms, implants and IUCD. They also had knowledge on Natural Family planning (NFP). The injectables and pills were mostly preferred. Most women found the method of contraception they used to be effective.

Major factors associated with the use of contraceptives included availability and knowledge on the method, marital status, age, level of education, occupation, partner's support, cost and side effects.

\subsection{Recommendations}

- The health workers within the MCH clinic should not limit the health education only on the methods available in the facility but should include all family planning methods.

- The administration of the hospital should ensure continuous supply of the contraceptives to avoid inconveniencing the clients.

- The Health workers should promote the use of nonhormonal contraceptives as they have less complication.

\section{Acknowledgement}

Uasin-Gishu Sub-County Hospital Administration for granting us the permission to conduct the research and the staff at the MCH/FP clinic for their support during the research data collection periods.

\section{References}

[1] Tsui A.O et al., 2011 Managing unplanned pregnancies in five countries: Perspectives on contraception and abortion decisions, Global Public Health.

[2] Timothy C. Okech, Nelson W. Wawire and Tom K. Mburu, 2011 Contraceptive use among women of reproductive age in Kenya's city slums, International journal of business and social science vol. 2 (1)

[3] E. K. Harrington, S. J. Newmann, M. Onono et al. 2012 "Fertility intentions and interest in integrated family planning services among women living with HIV in Nyanza Province, Kenya: aqualitative study," Infectious Diseases in Obstetrics and Gynaecology.

[4] Family Health Research Newsletter, 2007. Vol. 1 (1). USAID. Assessed from Family Health Research Newsletter, 2007. www.fhi360.org/sites/default/files/media/.../EFHR11global1.p df Assessment date: 15th march, 2105.

[5] Nwachukwu, IKE, Obasi, O. O. and Okpara, M 2008 Use of Modern Birth Control Methods Among Rural Communities in Imo State, Nigeria. African Journal of Reproductive Health, 12(1): 101-108.

[6] Imbareen Khan, Haleema. A, Hashmi, Zehra Naqvi, 2011 Awareness and practices of contraception, journal of surgery Pakistan, vol.16 (4) :182.

[7] Murerwa J. Mutiri ( 2012) Use of contraceptives among women of child bearing age, A case study of Kibera slums, Health policy and Planning, Nairobi province.

[8] Obugbenga-Bello AI, Abodonrim Oh, Adeoni S A.A, (2009), Contraceptive practices among women in rural communities in South-Western Nigeria, Journal of biosocial science.

[9] Orems, George, (2002) Determinants of contraceptive choice among women in South Africa, Family planning perspectives. 
[10] Republic of Kenya, Demographic Health Survey of 2008, Nairobi, Government printer, 2009.

[11] Teresa Saliku, Rhoune Ochako and chimaraokelzugbara, Use of contraceptive among women in Nairobi, Kenya APHRC, 2011.
[12] Wangui Joseph, Central Kenya Contraceptive use, Journal of health services research and policy, 2012. 